Evaluación ciudadana del primer año de

pp. $66-81$ gobierno de francisco flores

\title{
ENCUESTA
}

\section{Evaluación Ciudadana del Primer año de Gobierno de \\ Francisco Flores}

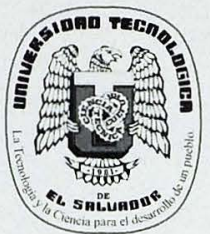

Centro de Investigación de la $\mathrm{O}_{\text {pinión }}$

Dública Salvadoreña (CIODS)

LA MUESTRA FUE DISTRIBUIDA DE ACUERDO A LAS SIGUIENTES VARIABLES:

\begin{tabular}{|c|c|}
\hline \multicolumn{2}{|c|}{ DEPARTAMENTO } \\
\hline San Salvador & 28.83 \\
\hline Santa Ana & 8.66 \\
\hline San Vicente & 2.76 \\
\hline Usulután & 5.85 \\
\hline Ahuachapán & 4.93 \\
\hline San Miguel & 8.38 \\
\hline Sonsonate & 6.77 \\
\hline La Unión & 4.93 \\
\hline La Paz & 4.61 \\
\hline La libertad & 9.72 \\
\hline Morazán & 4.93 \\
\hline Chalatenango & 3.41 \\
\hline Cabañas & 2.63 \\
\hline Cuscatlán & 3.59 \\
\hline \multicolumn{2}{|c|}{ SEXO } \\
\hline Femenino & 50.30 \\
\hline Masculino & 49.70 \\
\hline \multicolumn{2}{|c|}{ ESTADO CIVIL } \\
\hline Soltero & 44.59 \\
\hline Casado & 36.16 \\
\hline Acompañado & 13.27 \\
\hline Divorciado & 2.49 \\
\hline Viudo & 2.86 \\
\hline Sin Opinión & 0.64 \\
\hline
\end{tabular}

\begin{tabular}{|lr|}
\hline & EDAD \\
\hline $18-25$ & 26.07 \\
$26-35$ & 26.21 \\
$36-45$ & 20.59 \\
$46-55$ & 14.33 \\
56 ó mas & 12.39 \\
No contesto & 0.41 \\
\hline
\end{tabular}

\begin{tabular}{|lr|}
\hline & ESCOLARIDAD \\
\hline Ninguna & 11.70 \\
$1^{\circ}$ a $3^{\circ}$ & 10.32 \\
$4^{\circ}$ a $6^{\circ}$ & 17.69 \\
$7^{\circ}$ a $90^{\circ}$ & 21.46 \\
Bachiller & 27.91 \\
Técnico & 3.36 \\
Universitario & 6.59 \\
No contesto & 0.97 \\
\hline
\end{tabular}

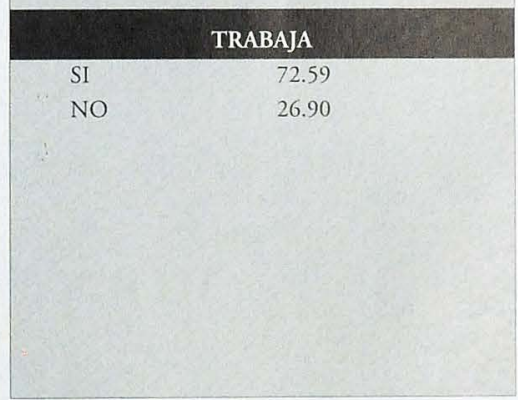


Evaluación ciudadana del primer año de

pp. $66-81$ gobierno de francisco flores

\section{FICHA TÉCNICA}

\section{INSTITUCION RESPONSABLE}

Universidad Tecnológica de El Salvador a través del Centro de Investigación de la Opinión Pública Salvadoreña.

\section{INSTITUCION PATROCINADORA}

Universidad Tecnológica de El Salvador

\section{PROYECTO}

XXIII Encuesta de Opinión Pública

\section{FECHA DE REALIZACION}

26,27 y 28 de Mayo de 2000

\section{OBJETIVO}

Evaluar la gestión del Gobierno del Presidente Francisco Flores durante su primer año de gobierno.

\section{AREAS DE EVALUACION}

Se investigó en las áreas económica, política y social.

\section{FINANCIAMIENTO}

Fondos destinados por la Universidad Tecnológica de El Salvador

\section{POBLACION}

$5,118,599$ habitantes.

Este dato para la encuesta de opinión pública, se tomó de acuerdo al V Censo de Población y IV de vivienda del Ministerio de Economía, Dirección General de Estadísticas y Censos, 1993.

\section{MUESTRA}

2,100

Se utilizó para el cálculo de esta muestra la fórmula para un diseño muestral probabilístico de poblaciones numerables finitas, la cual fue distribuida en los 14 departamentos y 90 municipios.

\section{BOLETAS VALIDAS}

\section{2,100}

\section{METODO}

Se urilizó el método de distribución por cuotas, asignándole a cada departamento y punto de evaluación una cantidad de boletas de a cuerdo al porcentaje de población correspondiente, seleccionándose los encuestados en forma aleatoria simple, ubicándolos en parques, colonias, mercados, negocios, etc.

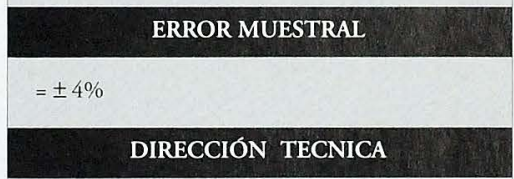

Ing. Nelson Zárate Sánchez 
El sondeo de opinión pública realizado por el CIOPS de la Universidad Tecnológica, abordó el tema de la situación económica, política y social en el Salvador.

A las personas que se seleccionaron para conformar la muestra, se les mostró tres fichas, para que en cada una de ellas señalará el problema que según su criterio, es el más grave que enfrenta nuestro país.

Los resultados se pueden observar en las siguientes tablas:

\section{BLOQUE \#1}

\begin{tabular}{|l|r|}
\hline Todo Caro & 52.88 \\
\hline Educación & 8.11 \\
\hline Escasez de Agua & 4.61 \\
\hline Delincuencia Juvenil & 22.43 \\
\hline Transporte & 0.83 \\
\hline Secuestros & 10.04 \\
\hline Ninguna & 0.32 \\
\hline S.O. & 0.78 \\
\hline
\end{tabular}

\section{BLOQUE \#2}

\begin{tabular}{|l|r|}
\hline Salarios Bajos & 49.47 \\
\hline Salud & 10.32 \\
\hline Contaminación & 10.23 \\
\hline Delincuencia & 24.64 \\
\hline Estado de Carreteras & 2.40 \\
\hline Casinos & 1.52 \\
\hline Ninguno & 0.55 \\
\hline S.O. & 0.88 \\
\hline
\end{tabular}

\section{BLOQUE \#3}

\begin{tabular}{|l|r|}
\hline Desempleo & 60.25 \\
\hline Vivienda & 7.46 \\
\hline Deforestación & 6.86 \\
\hline Narcotráfico & 6.68 \\
\hline Corrupción & 13.4 \\
\hline Prostitución & 3.32 \\
\hline Ninguno & 0.78 \\
\hline S.O. & 1.24 \\
\hline
\end{tabular}

Los problemas señalados con mas frecuencia en cada uno de estos bloques tienen que ver con situaciones de carácter económico, el Desempleo ocupa el porcentaje más al to $60.25 \%$, seguido de Todo caro con el $52.88 \%$ y los Salarios bajos con el $49.47 \%$.

Comparando la evaluación realizada por el CIOPS en Noviembre de 1995, para evaluar la gestión del gobierno en turno los problemas que ocuparon los primeros lugares fueron: Todo Caro en primer lugar, Delincuencia en segundo lugar, Desempleo en tercer lugar, las Maras en cuarto lugar y la Corrupción en quinto lugar. Nos da como resultado que en ese año dos de los problemas económicos actuales ya existían, apareciendo en ésta evaluación la problemática los Salarios Bajos.

En el siguiente cuadro se comparan los resultados de los primeros 100 dias del presidente Francisco Flores:

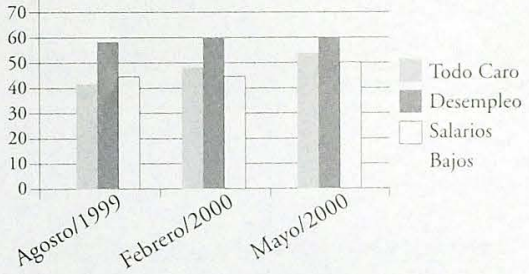

Podemos observar que el Desempleo se ha mantenido en este periodo.- Los Salarios Bajos y Todo Caro aumentaron

Para el $68.45 \%$ de la población el gobierno no está resolviendo los problemas que mencionó y de estas personas que contestaron $\mathrm{NO}$ el $32.57 \%$, consideran que no lo resuelven porque al gobierno no le importan los problemas del pueblo, el $30.08 \%$ consideran que es Incapacidad para Gobernar, el $26.92 \%$ consideran que es poco el tiempo que tienen de gobernar.

Se les presentaron 3 bloques para que opinaran sobre las acciones que el gobierno le está dando mayor atención. 
LOS RESULTADOS SON LOS SIGUIENTES:

\section{BLOQUE \#1}

\begin{tabular}{|l|r|}
\hline Combate a la Delincuencia & $30.72 \%$ \\
\hline Privatizaciones & $43.99 \%$ \\
\hline Control de la inflación & $3.78 \%$ \\
\hline Maquilas & 7.74 \\
\hline Ninguna & $10.36 \%$ \\
\hline S.O & $3.41 \%$ \\
\hline
\end{tabular}

\section{BLOQUE \#2}

\begin{tabular}{|l|r|}
\hline Proyectos de Salud & $21.05 \%$ \\
\hline Const. y reparac. de carreteras & $38.32 \%$ \\
\hline Creación de fuentes de trabajo & $10.27 \%$ \\
\hline Apoyo al sector agropecuario & $10.59 \%$ \\
\hline Ninguna & $15.02 \%$ \\
\hline S.O. & $4.74 \%$ \\
\hline
\end{tabular}

\section{BLOQUE \#3}

\begin{tabular}{|l|r|}
\hline Apoyo a la Educación & $37.77 \%$ \\
\hline Protección al Medio Ambiente & $12.16 \%$ \\
\hline Construcción de Vivienda & $14.23 \%$ \\
\hline Apoyo a la Pequeña Empresa & $10.50 \%$ \\
\hline Ninguna & $19.53 \%$ \\
\hline S.O. & $5.80 \%$ \\
\hline
\end{tabular}

Con relación a la situación económica del entrevistado comparándola con la del año pasado, el $45.32 \%$ consideró que la situación esta igual, el $39.11 \%$ manifestó que estaba peor y el $13.96 \%$ dijo que mejor. Si comparamos con la encuesta realizada en Agosto del año pasado, existe un incremento que lo podemos observar en el siguiente gráfico:

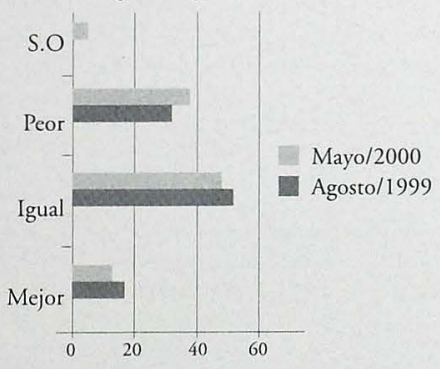

El año pasado el $50.56 \%$ consideraba la situación igual que el año anterior, el $33.37 \%$ lo consideraba peor $y$ el $15.49 \%$ lo considera mejor (Encuesta, CIOPS Agosto/99).

Las perspectivas de mejoras de la situación económica de la población, no son muy alentadoras, por la gestión que realiza en estos momentos el gobierno; el $65.41 \%$ de la población considera que la gestión actual no ayudará a mejorar su condición económica.

Con las medidas que el gobierno ha adoptado en relación a los impuestos del I.V.A. el $88.58 \%$ considera que este afectará su economía familiar.

Además, la percepción de la población sobre el incremento de la tarifa de los servicios básicos es notoria, el $69.92 \%$ observa que se ha incrementado el servicio de agua, el $80.15 \%$ el servicio de luz eléctrica, el $59.79 \%$ el servicio relefónico y el $44.27 \%$ el servicio de transporte.

Para el $64.81 \%$ de la población consideran que durante este año de gobierno no se han incrementado las fuentes de trabajo, y para $65.59 \%$ el presidente Francisco Flores no está cumpliendo lo que prometió en su campaña.

\section{La Población Evalúa el Trabajo del Presidente Francisco Flores.}

La calificación que la población da al presidente Francisco Flores en cuanto a su imagen actual, comparada con la que tenía durante la campaña política es la siguiente:

$49.38 \%$ considera que es igual, $27.96 \%$ peor y $17.37 \%$ mejor.

En cuanto al nivel de confianza que éste realiza el $55.60 \%$ manifiestan que tienen poca confianza, el $23.68 \%$ manifiestan que no tienen nada de confianza y el 15.43 manifestaron mucha confianza.

Para el $61.22 \%$ de la población las decisiones del presidente Francisco Flores ante la problemárica nacional no han sido acertadas.

Se le preguntó a la población que nivel de confianza le merece el actual gabinete de gobierno; mucha contestó $9.67 \%$, poca $41.87 \%$, nada el $25.70 \%$ y manifestaron que no conocían nada sobre actual gabinete de gobierno el $4.88 \%$. 


\section{ENCUESTA}

Para el $42.42 \%$ de la población el presidente Francisco Flores ha mejorado su estrategia de comunicación y el $48.60 \%$ manifestó que NO.

El $62.00 \%$ de la población considera que el presidente Francisco Flores no mantiene la misma popularidad que obtuvo durante su campaña política, el $30.48 \%$ considera que si la tiene.

En la encuesta realizada en Agosto/1999 el 61.18\% manifestó que el presidente no tenía la misma popularidad. Podemos observar que se ha incrementado con relación al mes de Agosto del año pasado en $0.82 \%$.

Sobre las características que la población observa que el presidente Francisco Flores posee, podemos resaltar :

Los ministerios que para la población están realizando un mejor trabajo son los siguientes: Educación con el $24.83 \%$, Salud $11.65 \%$, Obras Publicas $8.48 \%$, Ninguno $22.71 \%$, S.O. $13.04 \%$ y el resto de ministerios oscila entre $0.28 \%$ y $4.97 \%$.

La tendencia sobre el desempeño del trabajo que ha realizado durante el año de gobierno el presidente Francisco Flores la población evalúa entre regular y mala su gestión . El $77.20 \%$ de la muestra afirma lo expresado anteriormente.

\begin{tabular}{|l|c|c|c|c|}
\hline OPCION & SI & NO & N.S. & S.O. \\
\hline ACCESIBLE & 44.17 & 38.55 & 12.25 & 5.02 \\
\hline COMUNICATIVO & 55.14 & 28.97 & 11.05 & 4.84 \\
\hline SINCERO & 30.58 & 46.38 & 15.98 & 7.05 \\
\hline RESPETUOSO & 71.49 & 14.69 & 9.12 & 4.7 \\
\hline INTELUGENTE & 66.42 & 18.38 & 9.44 & 5.76 \\
\hline LIDERAZGO & 43.21 & 35.19 & 13.68 & 7.92 \\
\hline
\end{tabular}

Comparando con la encuesta realizada en agosto/ 1999 el resultado fue del 76.14\% que evaluó el trabajo entre regular y malo.

Y comparando con la encuesta de febrero/2000 el $77.10 \%$ lo evalúo también entre regular y malo.

La población cree que la situación del país no mejorará en los próximos 4 años ya que el $46.02 \%$ de manifestó que NO, el $42.42 \%$ manifestó que SI y el $11.56 \%$ no dio su opinión.
De acuerdo a lo manifestado por la población en cuanto a si votarían por Francisco Flores si las elecciones se repitieran, el $56.93 \%$ dijo que NO, el $31.18 \%$ dijo que SI y el $11.88 \%$ Sin Opinión.

\section{Población CUESTIONA MÉTOdo de ELECCIÓN DEL PRESIDENTE DE LA Asamblea LegisLativa.}

Para el $46.84 \%$ de la población, el proceso de elección del actual presidente de la Asamblea Legislativa no fue correcto, el $32.80 \%$ manifestó que si lo era y el $20.36 \%$ no dio su opinión. Se consultó a la personas que contestaron $\mathrm{NO}$ en esta pregunta sobre que partido político debería poseer la presidencia de la Asamblea Legislativa, el $61.55 \%$ manifestó que el FMLN, el $22.62 \%$ sin opinión, el $9.05 \%$ ARENA y el $5.90 \%$ PDC.

Sobre esta elección se consultó si la accitud mostrada por ARENA se vería afectada como partido, el $49.47 \%$ manifestó que SI, el $34.22 \%$ manifestó que NO y solo el $16.31 \%$ no dio su opinión. A las personas que contestaron que sí en ésta pregunta, el $47.58 \%$ consideran que le afectará perdiendo votos en las próximas elecciones.

El 54.63\% no esta de acuerdo que el PCN posea la presidencia de la Asamblea Legislativa, el $32.70 \%$ si lo está y el $12.67 \%$ no dio su opinión.

De acuerdo a lo manifestado por la población en cuanto a si votarían por Francisco Flores si las elecciones se repitieran, el $56.93 \%$ dijo que NO, el $31.18 \%$ dijo que SI y el $11.88 \%$ Sin Opinión.

\section{Población cuestiona método de ELECCIÓN DEL PRESIDENTE DE LA Asamblea Legislativa.}

Para el $46.84 \%$ de la población, el proceso de elección del actual presidente de la Asamblea Legislativa no fue correcto, el $32.80 \%$ manifestó que si lo era y el $20.36 \%$ no dio su opinión. Se consultó a la personas que contestaron $\mathrm{NO}$ en esta pregunta sobre que partido político debería poseer la presidencia de la Asamblea Legislativa, el $61.55 \%$ manifestó que el FMLN, el $22.62 \%$ sin opinión, el $9.05 \%$ ARENA y el $5.90 \%$ PDC.

Sobre esta elección se consultó si la accitud mostrada por ARENA se vería afectada como partido, el $49.47 \%$ manifestó que SI, el $34.22 \%$ manifestó que NO y sólo el $16.31 \%$ no dio su opinión. A las personas que 


\section{Evaluación del Primer año de Gobierno de Francisco Flores}

contestaron que sí en ésta pregunta, el $47.58 \%$ consideran que le afectará perdiendo votos en las próximas elecciones.

El 54.63\% no esta de acuerdo que el PCN posea la presidencia de la Asamblea Legislativa, el 32.70\% si lo está y el $12.67 \%$ no dio su opinión.

Para el $47.49 \%$ de la población no considera que el FMLN deba mantener la actitud de no ocupar ningún cargo dentro de la junta directiva de la Asamblea Legislativa, el 40.26\% considera que SI, el $12.25 \%$ no manifestó opinión.

Se mostró una ficha a la población para que opinara sobre las causas por las cuales el partido ARENA no ganó la mayoría de diputados en las pasadas elecciones, el resultado fue el siguiente:

\begin{tabular}{|l|c|}
\hline OPCION & $\%$ \\
\hline Perdida de confianza en el partido & 41.92 \\
\hline Desempeño del presidente de la república & 12.25 \\
\hline Candidatos no idoneos & 14.33 \\
\hline Diferencias internas en el partido & 6.96 \\
\hline Aumento de simpatizantes de otros partidos & 6.86 \\
\hline Mejores propuestas políticas de otros partidos & 7.14 \\
\hline S.O. & 10.55 \\
\hline
\end{tabular}

\section{Proyección de Votos.}

Considerando la tendencia actual y las condiciones económicas, políticas y sociales del país se consultó a la población si de mantenerse estas condiciones, que partidos políticos tiene mayores probabilidades de ganar las elecciones del 2004, los resultados fueron los siguientes:

\begin{tabular}{|l|c|}
\hline PARTIDO & $\%$ \\
\hline ARENA & 18.15 \\
\hline FMLN & 53.66 \\
\hline PCN & 3.73 \\
\hline PDC & 1.11 \\
\hline PAN & 0.18 \\
\hline NINGUNO & 3.73 \\
\hline S.O. & 19.44 \\
\hline
\end{tabular}




\section{ENCUESTA}

\section{Disthinución Depahtamental}

\begin{tabular}{|c|c|}
\hline San Salvador & 28.83 \\
\hline Santa Ana & 8.66 \\
\hline San Vicente & 2.76 \\
\hline Usulután & 5.85 \\
\hline Ahuachapán & 4.93 \\
\hline San Miguel & 8.38 \\
\hline Sonsonate & 6.77 \\
\hline
\end{tabular}

\begin{tabular}{|l|r|}
\hline La Unión & 4.93 \\
\hline La Paz & 4.61 \\
\hline La libertad & 9.72 \\
\hline Morazán & 4.93 \\
\hline Chalatenango & 3.41 \\
\hline Cabañas & 2.63 \\
\hline Cuscatlán & 3.59 \\
\hline
\end{tabular}
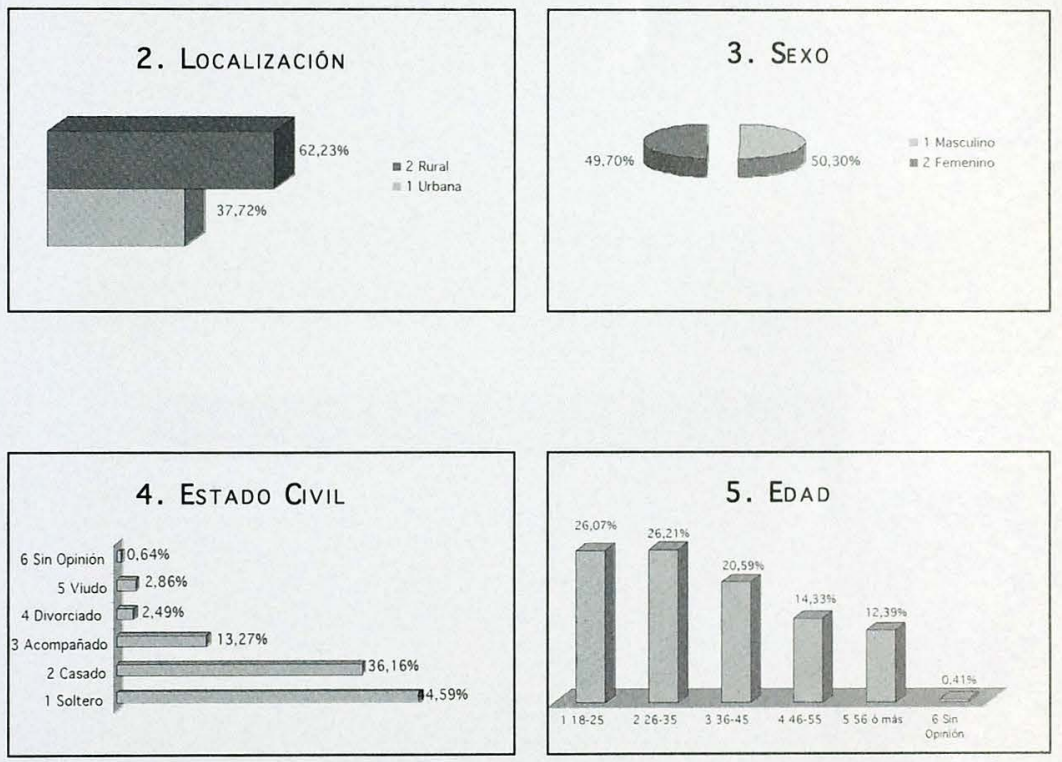

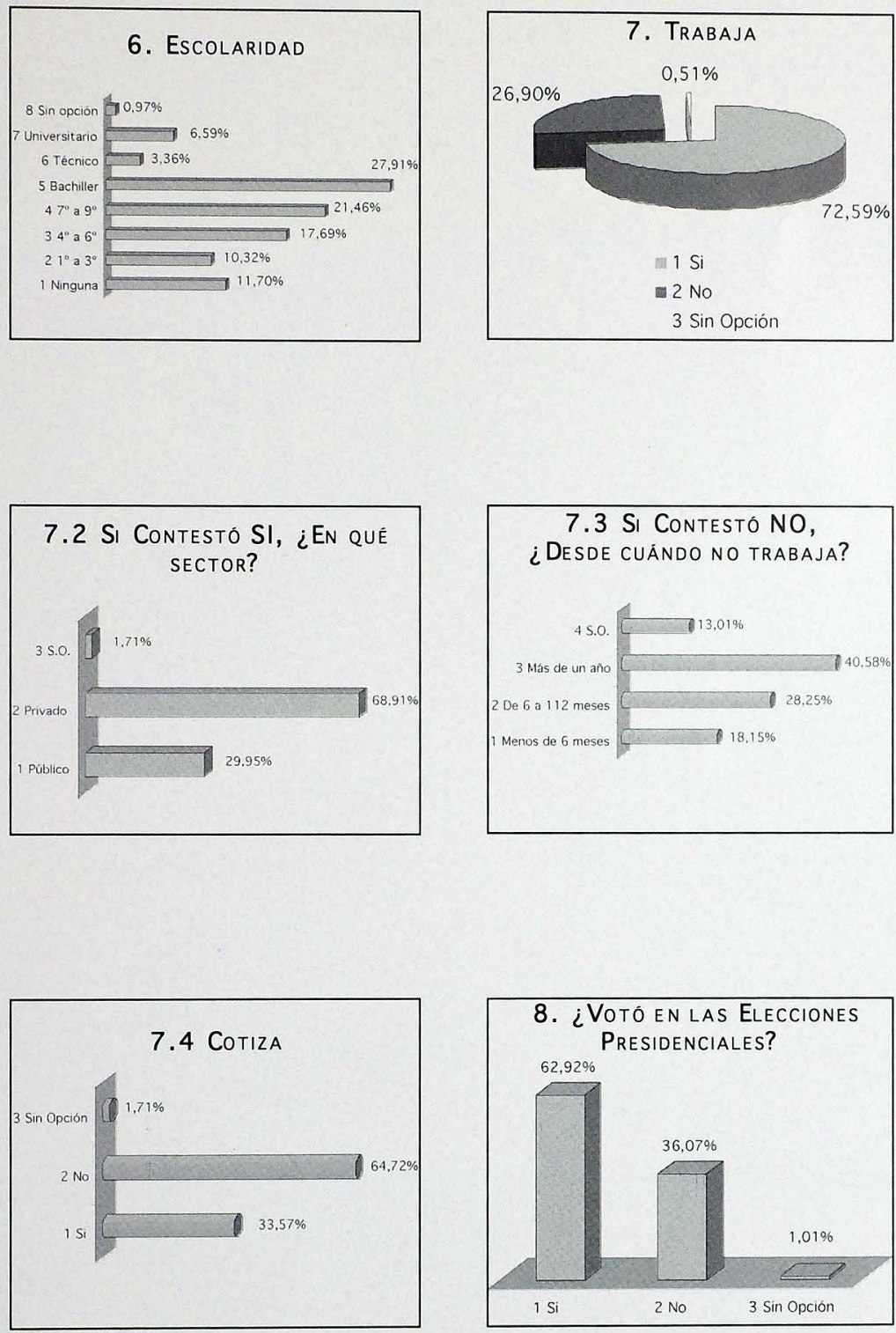


\section{Preguntas}

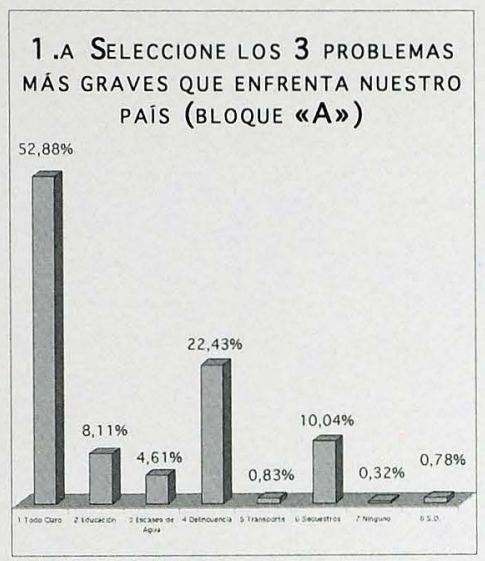

1.b Seleccione los 3 problemas MÁs gRAVES QUE ENFRENTA NUESTRO PAIS (BLOQUE «B»)

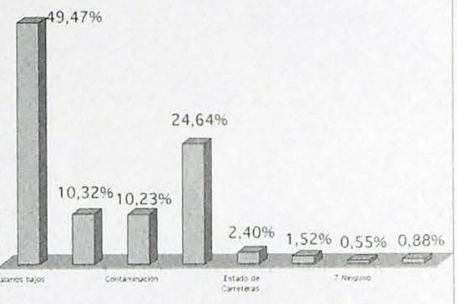

1.c Seleccione los 3 problemas MÁS GRAVES QUE ENFRENTA NUESTRO PAIS (BLOQUE «A»)

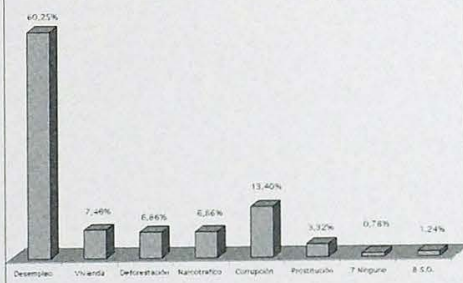

2. ¿Creé que el Gobierno está RESOLVIENDO LOS PROBLEMAS QUE MENCIONÓ?

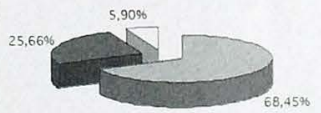


Evaluación ciudadana del primer año de gobierno de francisco flores

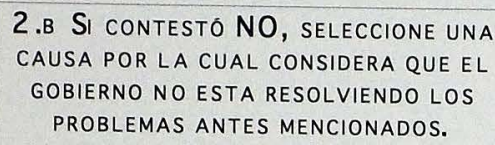

\begin{tabular}{|c|c|}
\hline Concento & $\%$ \\
\hline 1. Poco reimpo de gobernar & 26.92 \\
\hline 2. Incapacidad para gobernar & 30.08 \\
\hline 3. No le importan los problemas del pueblo & 32.57 \\
\hline 4. No lo dejan los otros partidos políticos & 7.20 \\
\hline 5. S.O. & 3.23 \\
\hline
\end{tabular}

3. B De LAS Siguientes acciones, ¿A CUÁles CREÉ QUE EL GOBIERNo LE BRINDA MAYOR ATENCIÓN? (BLOQUE

B)

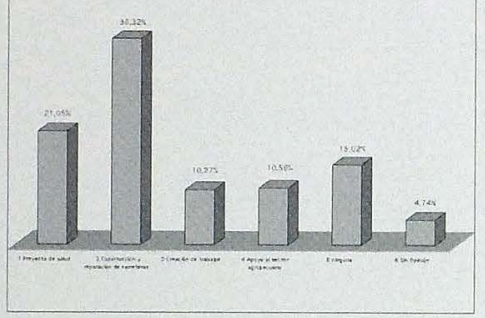

4. En este momento ¿Cómo está

LA SITUACIÓN ECONÓMICA DE SU HOGAR EN COMPARACIÓN CON EL AÑO PASADO?

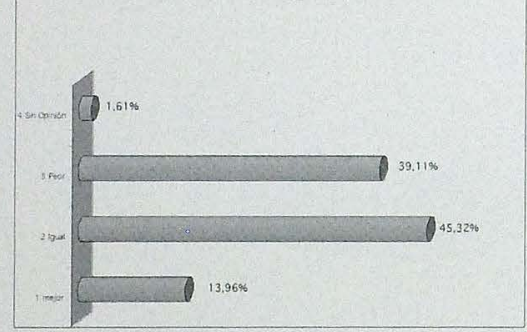

3.A DE LAS Siguientes aCcIONE, ¿A CUÁles CREE QUe el Gobierno le BRINDA MAYOR ATENCIONN?

(BLoque A)

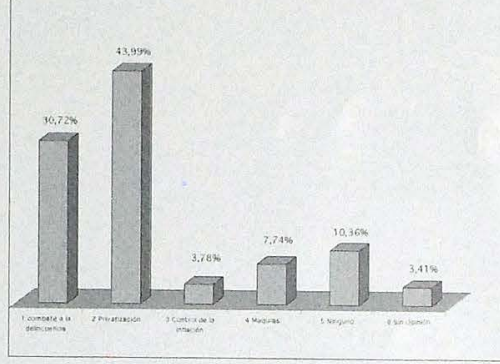

3.c De Las siguientes acciones, ¿Cuáles creéque el gobierno le BRINDA MAYOR ATENCIÓN?

(BLOQUe C)

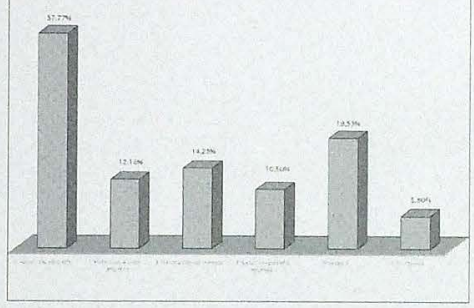

5. ¿Considera que la gestión del Gobierno actual MEJORARÁ SU CONSICION ECONOMICA?

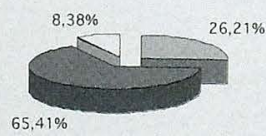

is $1 \mathrm{Si}$

3 Sin Opinión 


\section{ENCUESTA}

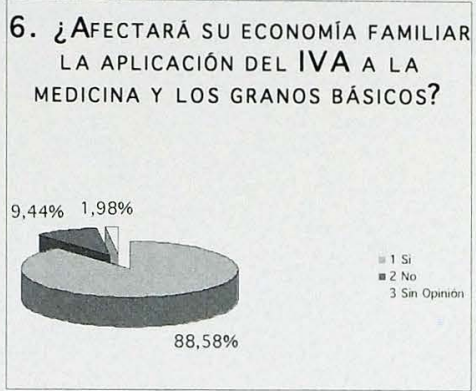

8. ¿TiEne familiares o conocidos DESPEDIDOS DURANTE EL ÚLTIMO AÑO?

$78.82 \%$
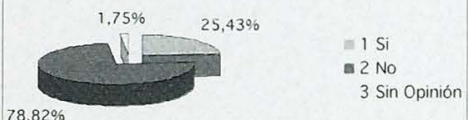

9. ¿Considera que durante el Gobierno del Presidente Francisco FLORES SE HAN INCREMENTADO LAS FUENTES DE TRABAJO?
3 Sin Opinión

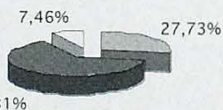

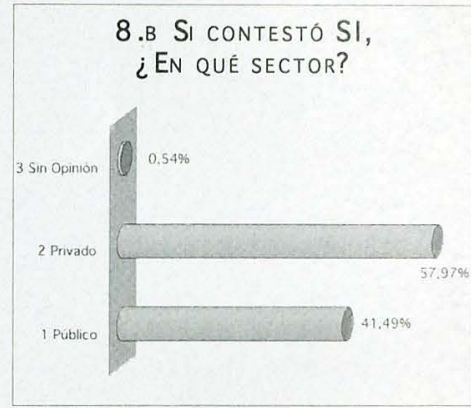

7. De Los Siguientes SERVICIOS BÁSICOS, ¿CUÁLES HAN SUFRIDO INCREMENTO EN EL ÚLTIMO AÑO?

\begin{tabular}{|l|l|l|l|}
\hline Servicio & Si & 1 I o & Sin \\
\hline Agua & $69.92 \%$ & $23.49 \%$ & $6.59 \%$ \\
\hline Luz & $80.15 \%$ & $14.92 \%$ & $4.93 \%$ \\
\hline Telefono & $59.79 \%$ & $27.82 \%$ & $12.39 \%$ \\
\hline Transporte & $44.27 \%$ & $46.52 \%$ & $9.21 \%$ \\
\hline
\end{tabular}

8. B Si Contestó Si, ¿EN QUÉ SECTOR?

10. ¿Creé que el Presidente FRANCISCO FLORES ESTÁ CUMPLIENDO LO QUE PROMETIÓ EN SU CAMPAÑA ELECTORAL?

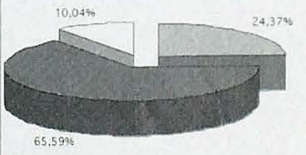

$1 \mathrm{Si}$ 2 No $3 \operatorname{Sin}$ Opinión 
Evaluación ciudadana del primer año de

pp. $66-81$ gobierno de francisco flores

11. Con relación a la imagen del Presidente Francisco Flores DURANTE LA CAMPAÑA POLITICA, ¿Cómo la claifica actualmente?

\begin{tabular}{|l|r|}
\hline \multicolumn{1}{|c|}{ Concepto } & $\%$ \\
\hline 1. Mejor & 17.37 \\
\hline 2. Igual & 49.38 \\
\hline 3. Peor & 27.96 \\
\hline 4. Sin opinión & 5.30 \\
\hline
\end{tabular}

13. ¿Considera que las decisiones del Presidente Francisco Flores HAN SIDO ACERTADAS ANTE LA PROBLEMÁTICA NACINAL?

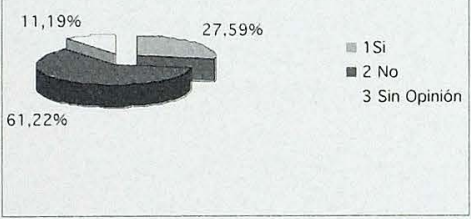

15. ¿CReé Que el Presidente Francisco Flores ha MEJORAdO SU ESTRATEGIA DE COMUNICACIÓN CON LA POBLACION?

$48,60 \%$

$8,98 \%$

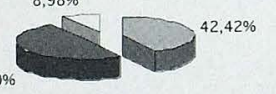

- $1 \mathrm{Si}$

a 2 No

3 Sin Opinión
16. ¿Considera Que el

Presidencia Francisco Flores MANTIENE LA MISMA POPULARIDAD QUE OBTUVO DURANTE SU CAMPAÑA POLITICA?

4. ¿QUÉ NIVEL DE CONFIANZA LE MERECE EL ACTUAL GABINETE DE Gobierno?
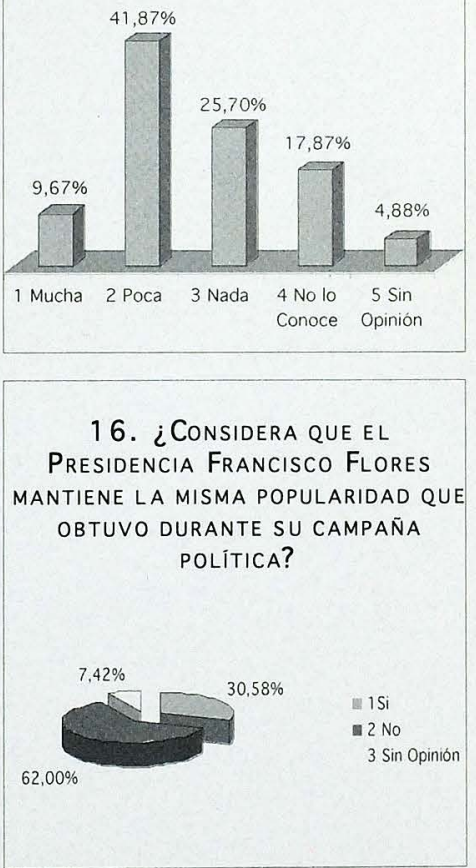


\section{ENCUESTA}

17. DE LAS SIGUIENTES CARACteristicas ¿CuÁles Posee El Presidente Francisco Flores en el DESEMPEÑO DE SUS FUNCIONES?

\begin{tabular}{|l|c|r|r|r|}
\hline Servicio & \multicolumn{1}{c}{ Si } & \multicolumn{1}{c}{1 lo } & \multicolumn{1}{c|}{ Sin } & S. O. \\
\hline Accesible & $44.17 \%$ & $38.55 \%$ & $12.25 \%$ & $5.02 \%$ \\
\hline Comunicativo & $55.14 \%$ & $28.97 \%$ & $11.05 \%$ & $4.84 \%$ \\
\hline Sincero & $30.58 \%$ & $46.38 \%$ & $15.98 \%$ & $7.05 \%$ \\
\hline Respetuoso & $71.49 \%$ & $14.69 \%$ & $9.12 \%$ & $4.70 \%$ \\
\hline Inteligente & $66.42 \%$ & $18.38 \%$ & $9.44 \%$ & $5.76 \%$ \\
\hline Liderazgo & $43.21 \%$ & $35.19 \%$ & $13.68 \%$ & $7.92 \%$ \\
\hline
\end{tabular}

18. ¿CuÁl considera Que ES EL objetivo del Presidente Francisco

FLORES AL PRESENTAR EN CADA DEPARTAMENTO LAS INVERSIONES QUE EL GOBIERNO REALIZA EN EL MISMO?

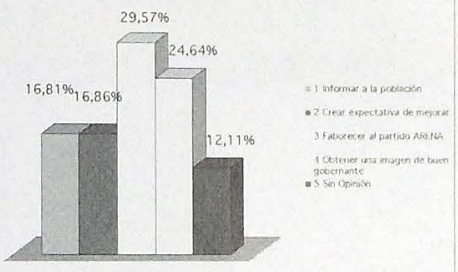

19. ¿Que Ministerio está REALIZANDO MEJOR TRABAJO EN LA GESTIÓN DEL PRESIDENTE FRANCISCO FLORES?

\begin{tabular}{|l|c|}
\hline 1. Educación & $24.83 \%$ \\
\hline 2.Salud & $11.65 \%$ \\
\hline 3. Trabajo & $4.97 \%$ \\
\hline 4. Justicia & $3.13 \%$ \\
\hline 5. Hacienda & $2.53 \%$ \\
\hline 6. Economia & $1.89 \%$ \\
\hline 7. Interior & $0.74 \%$ \\
\hline 8. Exteriores & $0.28 \%$ \\
\hline 9. Obras Públicas & $8.48 \%$ \\
\hline 10. Agricultura y Ganaderia & $2.03 \%$ \\
\hline 11. Madio Ambiente & $0.92 \%$ \\
\hline 12. Seguridad Pública & $2.81 \%$ \\
\hline 13. Ninguna & $22.71 \%$ \\
\hline 14. S. O. & $13.04 \%$ \\
\hline
\end{tabular}

20. ¿Cómo evalúa el trabajo Realizado por el Presidente Francisco FLORES DURANTE SU PRIMER AÑo de Gobierno?

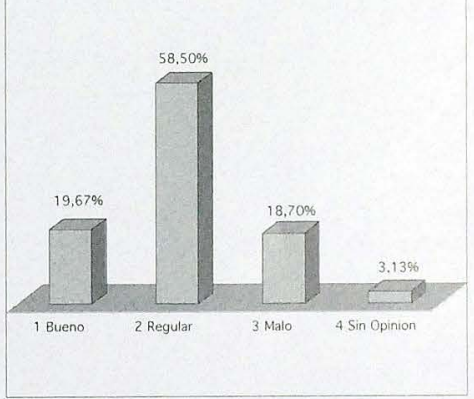


Evaluación ciudadana del primer año de

pp. $66-81$

gobierno de francisco flores

\section{Evaluación del Primer año de Gobierno de Francisco Flores}
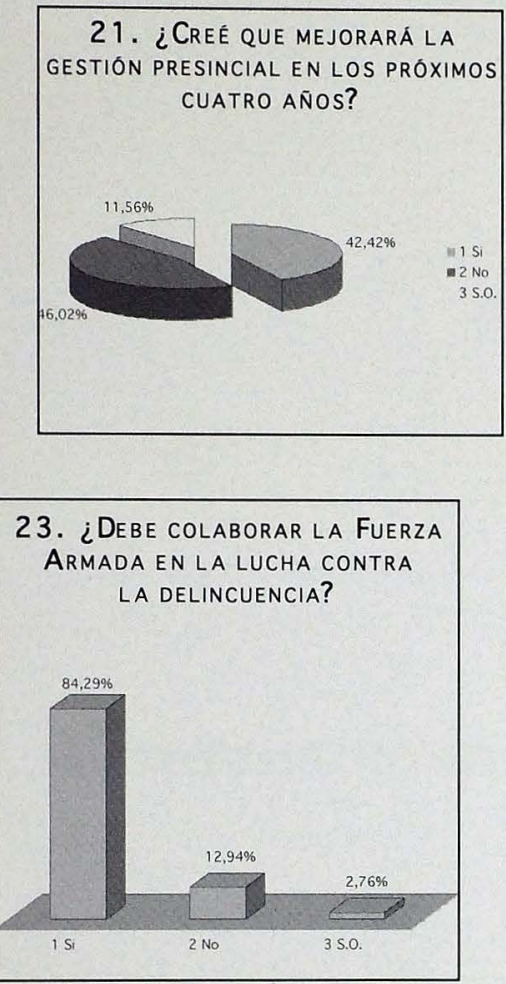

25. ¿CONSIDERA CORRECTO EL PROCESO DE ELECCIÓN DEL ACTUAL

Presidente de LA Asamblea LEGISLATIVA?

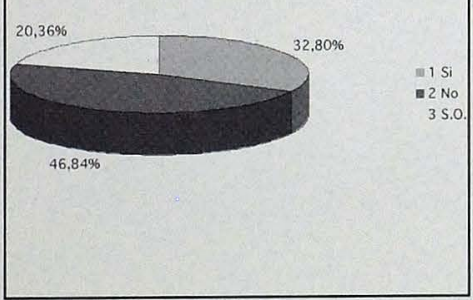

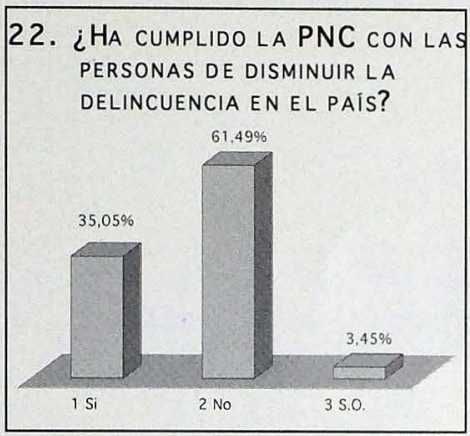

24. Si LAS ELECCIONES PRESINCIALES SE REPITIERAN HOY, ¿VOTARIA POR EL Lic. Francisco Flores?

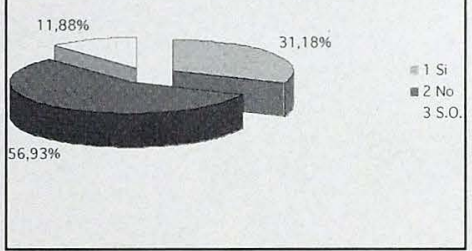

25.B SI CONTESto NO, ¿QUe PARTIDO POLITICO DEBERIA POSEER LA PRESIDENCIA DE LA ASAMBLEA LEGISLATIVA?

\begin{tabular}{|c|}
\hline ARENA \\
\hline $9.05 \%$ \\
\hline PDC \\
\hline $5.90 \%$ \\
\hline
\end{tabular}

\begin{tabular}{|c|}
\hline FMLN \\
\hline $61.55 \%$ \\
\hline PAN \\
\hline $0.88 \%$ \\
\hline
\end{tabular}

Sin Opinión

$22.62 \%$ 


\section{ENCUESTA}

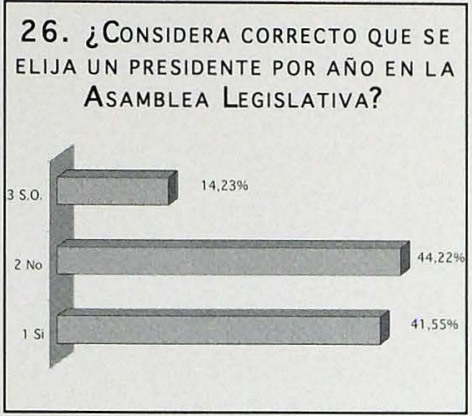

27 ¿AfECTARA AL PARTIDO ARENA LA ACTITUD MOSTRADA EN LA ELECCIÓN DEL PRESIDENTE DE LA ASAMBLEA LEgISLATIVA?
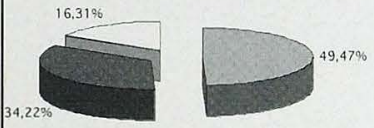

\begin{tabular}{|c|c|}
\hline & $\%$ \\
\hline 1. Poco teimpo de gobernar & 26.92 \\
\hline 2. Incapacidad para gobernar & 30.08 \\
\hline 3. No le importan los problemas del pueblo & 32.57 \\
\hline 4. No lo dejan los otros partidos políticos & 7.20 \\
\hline 5. S.O. & 3.23 \\
\hline
\end{tabular}

29. ESTÁ de ACUERdo QUE EL PCN POSEA LA PRESIDENCIA DE LA ASamblea legisLativa?

\section{B Si Contesto SI, ¿DE Qué MANERA LE AFECTARÁ?}

\begin{tabular}{|l|r|}
\hline \multicolumn{1}{|c|}{ Concepto } & $\%$ \\
\hline 1. Perdida de Popularidad & 31.28 \\
\hline 2. Descontento entre sus dirigentes & 20.39 \\
\hline 3. Pérdida de votos en la próximas elecciones & 47.58 \\
\hline 4. Sin opinión & 0.74 \\
\hline
\end{tabular}

26. B Si contesto SI, ¿Que PARTIDO DEBERIA OBTENER LA PRÓXIMA PRESIDENCIA DE LA ASAMBLEA LegISLATIVA?

ARENA
$23.61 \%$
PDC

FMLN

$41.57 \%$

PAN

$8.09 \%$ $1.66 \%$
$25.06 \%$ 
Evaluación ciudadana del primer año de

30. ¿A QUé atribuye QUe el PARTido

ARENA NO GANÓ LA MAYORIA DE DIPUTADOS EN LAS PASADAS ELECCIONES?

\begin{tabular}{|l|r|}
\hline \multicolumn{1}{|c|}{ Concepto } & \multicolumn{1}{c|}{$\%$} \\
\hline 1. Perdida de confianza en el partido & 41.92 \\
\hline 2. Desempeño del Presidente de la República & 12.25 \\
\hline 3. Candidatos no idóneos & 14.33 \\
\hline 4. Diferencias internas en el partido & 6.96 \\
\hline 5. Aumento de simpatizantes de otros partidos & 6.86 \\
\hline 6. Mejores propuestas políticas de otros partidos & 7.14 \\
\hline 7. S.O. & 10.55 \\
\hline
\end{tabular}

32. ¿QUe PARTIDO POLITICO TIENE MAYORES PROBABILIDADES DE GANAR LA Presidencia de la República para las ELECCIONES DEL 2004?

\begin{tabular}{|l|r|}
\hline \multicolumn{1}{|c|}{ Partido } & \multicolumn{1}{c|}{$\%$} \\
\hline 1. ARENA & 18.15 \\
\hline 2. FMLN & 53.66 \\
\hline 3. PCN & 3.73 \\
\hline 4. PDC & 1.11 \\
\hline 5. PAN & 0.18 \\
\hline 6. Ninguno & 3.73 \\
\hline 7. S.O. & 19.44 \\
\hline
\end{tabular}

31. ¿CONSidera QUe el FMLN debe MANTENER LA ACTITUD DE NO OCUPAR NINGÚN CARGO DENTRO DE LA JUNTA DiReCTIVA DE LA Asamblea

$$
\text { LegisLativa? }
$$

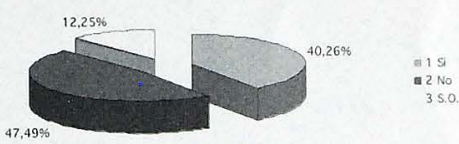

